\title{
Reliability and validity of pelvic mobility measurement using a cushion sensor in healthy adults
}

\author{
Seung-Hwa Jung ${ }^{(1)}$, Su-Kyeong Kim ${ }^{(1)}$, Ji-Hyun Lee ${ }^{(1)}$, Soo-Ih Choi ${ }^{(\infty)}$, Dae-Sung Park ${ }^{(1)}$ \\ Department of Physical Therapy, Konyang University, Daejeon, Republic of Korea
}

Objective: To prevent low back pain, an objective evaluation tool to evaluate pelvic mobility and exercise to improve the flexibility of the lumbar region is needed. The purpose of this study was to compare the results of pelvic mobility measurements using the Wii Balance Board (WBB) and Sensbalance Therapy Cushion (STC), evaluate the usefulness of the STC as a tool for measuring pelvic mobility.

Design: Cross-sectional study.

Methods: Fifty healthy subjects participated in this study. The subjects performed pelvic mobility range, proprioception, reaction time and reach of the arm using the STC. The pelvic movement parameter was measured two times to determine the intra-rater reliability. To measure the correlation between lumbar muscle tension and pelvic mobility, Myovision was used to measure tension of L4, L5 level erector spinae muscle. Correlations between measured variables were checked to determine the validity of the pelvic mobility assessment tool.

Results: STC showed high test-retest reliability in pelvic tilt measurement and reaching task [intraclass correlation coefficients $(3,1)=0.804-0.915]$. The relationship between WBB and STC showed a significant positive correlation with the pelvic tilt and reaching task $(p<0.05)$. Posterior tilt and erector spinae activation (Lt. L5) showed a significant negative correlation $(p<0.05)$. Left, right tilt and erector spinae activation (L5) showed a significant negative correlation $(p<0.05)$.

Conclusions: This study confirmed the advantages of the STC and found efficiency as an objective measuring device of pelvic mobility.

Key Words: Movement, Pelvis, Sitting position

\section{Introduction}

With the development of computer science and the economy, the working and academic environment has changed, and the time spent on assuming an incorrect sitting posture compared to performing dynamic physical activity has increased in the world of modern people today. In the sitting position, the physical burden put onto the waist is 7 times greater than when lying down, and more than 3 times greater than when standing, and a habitual assumption of an incorrect posture disproportionately disperses the weight, resulting in producing greater strains on the body [1]. As a re- sult, the incidence of low back muscle tension and lower back pain continues to increase, and the age of incidence is gradually decreasing [2].

Anatomically, the cervical and lumbar spine have an anterior curvature, the thoracic spine has a posterior curvature, and since the vertebrae and pelvis have a complex connection the position of the pelvis in the sitting position has a significant effect on the spine [3]. In general, the position of the pelvis can be used to measure problems of the spine and surrounding joints [4]. Deformation of the pelvic position occurs through various postural compensation for neuromusculoskeletal disorders [5], and changes in the pelvic po-

Received: 24 April, 2020 Revised: 26 May, 2020 Accepted: 29 May, 2020

Corresponding author: Dae-Sung Park (ORCID https://orcid.org/0000-0003-4258-0878)

Department of Physical Therapy, Konyang University, 158 Gwanjeodong-ro, Seo-gu, Daejeon 35365, Republic of Korea

Tel: 82-42-600-8456 Fax: 82-41-600-1098 E-mail: daeric@konyang.ac.kr

(a) This is an Open-Access article distributed under the terms of the Creative Commons Attribution Non-Commercial License (http://creativecommons.org/licenses/ by-nc/4.0) which permits unrestricted non-commercial use, distribution, and reproduction in any medium, provided the original work is properly cited

Copyright (๑) 2020 Korean Academy of Physical Therapy Rehabilitation Science 
sition through prolonged postural compensation cause various musculoskeletal disorders [6].

When the position of the pelvis is deformed, the pelvis goes into posterior pelvic tilt as a compensatory method of preventionting the trunk from being pushed posteriorly, resulting in a slumped posture. The left and right slopes of the pelvis appearing in the coronal plane creates an asymmetrical pressure on both cheeks and can cause spinal flexion. The abnormal posture caused by the above secondary problems is reduced flexibility due to tension of the spinal cord, lumbar fascia, ligaments, and articular pockets, and tension of the hip flexors and flexors, as well as imbalance of the body. It is a cause of low back pain and therefore, appropriate prevention is necessary [7].

Among the pelvic structures, the sacrotuberous ligament provides balance and stability of the pelvis. According to the fascial meridian theory, the vertebral iliac fascia coexists in the posterior superficial back line path of the sacroiliac ligament, and accordingly, pelvic mobility limitation is related to lumbar muscle activity [8]. According to previous studies, balanced muscle activity of the hip and torso is important for the stability of the pelvis and the balance of the body and that the movement of the lumbo-pelvic can elicit a balanced activity of the lumbar muscles and the muscles around the hip joint [9]. Therefore, it can be said that evaluating pelvic mobility is necessary to quickly identify problems with the pelvis and to properly prevent back pain.

Objective methods used to evaluate the lumbar region index include X-ray imaging, depth calipers, and motion analysis systems. Through X-ray imaging, anatomical evaluation and pelvic orientation can be confirmed using vertebra-pelvic parameters such as pelvic incidence, pelvic tilt, and sacroiliac tilt [10]. The Depth Caliper and Meter Stick, which is used to measure the pelvic tilt angle of a normal adult during standing, can define front and rear tilt by measuring the height difference between the anterior superior iliac spine and posterior superior iliac spine as an angle value [11].

Since the above measuring equipment is used during static standing positions, it is difficult to obtain a dynamic measurement values, such as pelvic mobility, in the sitting position. Conversely, a three-dimensional motion capture system (Vicon Camera, MX-T40; Vicon, Oxford, UK) can be used to analyze kinematic motion characteristics, which is expensive equipment and is difficult to use in a general treatment room. As an evaluation tool that can compensate for these limitations, pelvic movement ability can be measured with an objective value using the Wii Balance Board (WBB) or Sensbalance Therapy Cushion (STC).

WBB is a device that can measure the center of gravity and body pressure by installing an analog pressure sensor on the corner of a square plate [12]. WBB is used not only to measure the static balance ability in a standing position by recording the input pressure center, but also to perform a task using pelvic tilt in a sitting state, that is, using joint motion $[12,13]$.

By using STC visual feedback, you can exercise, train, and treat through a software such as games, record the user's movements in detail, and provide information on body stability, reaction speed, and potential imbalance. The collected data has the advantage of being able to perform comparative analysis because cumulative statistics and results are displayed instead of instant test results through computer software [14]. STC could save a lot of money and effort compared to the other equipment mentioned above, and will be able to provide objective information to the therapist and subject. However, reliability and validity studies as a pelvic mobility measurement device in a sitting position to prevent back pain in healthy adults have not been conducted. Therefore, in this study, the reliability of the STC was measured and the results of the pelvic mobility measurement using WBB and STC were compared to confirm the usefulness of the STC as a pelvic mobility measurement tool. This study was also conducted to confirm if there is a correlation that exists between the pelvic mobility variables.

\section{Methods}

\section{Participants}

The G* power (version 3.1.9.2; Heinrich-Heine-Universität, Düsseldorf, Germany) was used to calculate the study subjects. According to the results of the previous study, the sample size calculated by the test power ( $\rho$ ) of 0.95 and the significance level of 0.05 was 46 , and considering the potential for dropouts, 50 healthy adults over 20 years old were recruited [15]. The selection criteria were those who have not experienced low back pain in the past 3 months, those who tested negative on the Thomas test, and those who were negative for lower limb lift results. Those with experience of low back pain or those with radiation pain, those with a numerical pain scale of 3 or higher for their current low back pain, and those with positive reactions to the hip nerve lesion on the lower limb examination were excluded. All subjects provided their voluntary consent after hearing the explan- 
ation of the purpose and method of the study, and were conducted under the deliberation (IRB No. KYU-2019-333-01) of the Institutional Bioethics Committee of Konyang University.

\section{Experiment method}

\section{Measuring tools}

(1) Static muscle activity evaluation

For static surface electromyography, the Myovision EMG 4000 (PBI/myovision, San Carlos, CA, USA) was used. Static surface electromyography is a non-invasive device with five electrodes attached to two wireless joysticks. The Myovision is designed to record the level of muscle activity around the spine. The absolute value of the EMG signal is quantified. The actual data are measured in microvolts $(\mathrm{mV})$ and the tension level of the muscle is expressed in a bar-shaped stress score and recorded on a monitor [16]. A frequency filter of $20-500 \mathrm{~Hz}$ was used and the scanning range was 0.08 to $200 \mathrm{mV}$ [17]. The Myovision's intra-inspector test-retest reliability is high, with intraclass correlation coefficients $(\mathrm{ICC})=0.81-0.86$ [18].

\section{(2) Wii Balance Board}

The WBB (Nintendo, Kyoto, Japan) and Balancia software (Ver. 2.0; Mintosys, Seoul, Korea) were used as golden standard equipment to verify validity.

The WBB, manufactured for the purpose of home-use game machine, is equipped with four load cells of one axis on each corner in a rectangular shape, and is a device that can measure weight and body pressure center points, and can be easily used for clinical purposes. The WBB is a useful evaluation tool that has been verified as a balance measurement tool in standing posture, and the intra-inner reliability is as high as ICC $=0.92-0.98$, and the Balancia software used together also has a reliability of $\mathrm{ICC}=0.79-0.96$, and a validity of $\mathrm{ICC}=0.85-0.96$. This is a useful evaluation tool $[12,19]$.

\section{(3) Sensbalance Therapy Cushion}

The STC (Sensamove Inc., Utrecht, Netherlands) is a circular cushion type device with a built-in pressure sensor, which can be connected to a computer by wire and the body pressure center point is displayed on the software monitor. Data storage and processing were stored through a computer's Pedalo-sensamove-balance-test pro software (Sensamove Inc.). Through this equipment, the pelvic range of motion, intrinsic acceptance and response time, and the level of movement of the body pressure center point were evaluated based on the pressure center point.

\section{Research procedure}

This study evaluated two pelvic movement abilities. First, the measurement of the surface muscle activity of the subject's spinal column was performed in a sitting position. Since the measureed values may be affected, measurements were made after the skin was disinfected with alcohol, and subjects were instructed not to speak during the measurement. The measurement reference point was the $2-\mathrm{cm}$ point outside the 4th lumbar vertebrae, and the spinal cord muscle activity at the 3rd and 5th lumbar vertebrae (L3, L5 level) above and below the reference point was measured (Figure 1).

Next, to measure three variables related to pelvic movement ability and arm stretch task, the WBB was placed first on a flat table and STC was placed on the center of the stomach. Subjects were allowed to sit comfortably in the center of the STC, and a wooden box at appropriate height was placed on the sole of the foot so that the legs could be flexed to $90^{\circ}$. First, the range of motion of the pelvis was conducted with the monitor covered to block any potential visual feedback. Subjects were instructed to limit the movement of the trunk and to tilt the pelvis forward, backward, left, and right using the movement of the pelvis as much as possible. Second, the intrinsic acceptance sensory test was conducted by the subject directly checking the pressure center point output on the monitor. In the first attempt, the target point was printed on the monitor and the target point was reached. Immediately on the second attempt, the pressure center point output to the monitor disappeared and the same

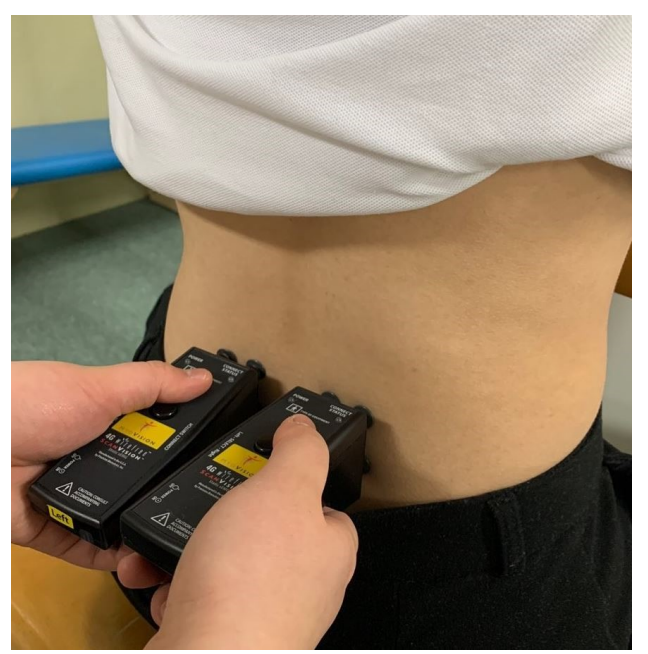

Figure 1. Surface muscle activity using the Myovision (L4, L5 level). 
target point was displayed. The subject completed the measurement and recorded the error up to the target point by using the intrinsic accommodation sense of the pelvic region to stop and hold it when it was considered that the target point was reached.Third, the reaction time test was traced as quickly as possible to the target point displayed on the monitor 4 seconds after the test start button was pressed. At this time, the reaction time was recorded (Figure 2A).

Finally, the arm stretch task was performed. After setting the target point at $120 \%$ of the arm length, the arm was stretched in a comfortable position and directed to reach the target, and the change in pressure center was recorded when the body moved by extending the arm forward, left, and right (Figure 2B, C). Next, subjects were allowed to walk on the treadmill at a comfortable speed for 15 minutes, and immediately the second pelvic motion measurement (pelvic range of motion, intrinsic acceptance test, reaction time test) was conducted.

\section{Data processing}

This study analyzed the collected data using the PASW Statistics for Windows, Version 18.0 (SPSS Inc., Chicago, IL, USA). ICC were used to calculate the intra-rater reliability of the STC $[\operatorname{ICC}(3,1)]$ and $95 \%$ confidence interval was obtained. ICC values of less than 0.40 is considered poor (poor), 0.4-0.6 is fair (fair), 0.60-0.75 is good (good), and greater than 0.75 is considered as excellent [20]. In order to determine the simultaneous validity of pelvic mobility between STC and WBB, the linear relationship between the measurement variables was examined and expressed through the Pearson correlation coefficient. All statistical significance levels were set to $\alpha=0.05$.

\section{Results}

\section{General characteristics of study subjects}

A total of 50 subjects ( 29 men and 21 women) participated in this study, with the average age of $20.9 \pm 1.5$ years, average height of $165.1 \pm 7.2 \mathrm{~cm}$, and the average weight of $58.8 \pm$ $10.6 \mathrm{~kg}$. Left L3 and L5 static surface electromyography values were $6.2 \pm 3.8 \mathrm{mV}$ and $7.3 \pm 4.9 \mathrm{mV}$, respectively, and right L3 and L5 static surface electromyography values were $6.7 \pm$ $4.9 \mathrm{mV}$ and $7.4 \pm 4.9 \mathrm{mV}$, respectively. There were $47 \mathrm{sub}-$ jects who were right-handed, and three subjects who were left-handed.

\section{Intra-rater reliability}

Table 1 shows the results of the pelvic mobility test-retest of the subjects measured by the same tester using the STC. The test-retest results for the front slope, the rear slope, the left lateral slope, and the right lateral slope showed excellent agreement. The ICC for the front, rear, and right intrinsic perception was poor. The ICC for the anterior, posterior, left and right response times was poor to fair. The ICC according to the forward, left, and right stretch tasks was very good.

\section{Validity of the pelvic mobility evaluation}

Table 2 shows the results of the Pearson's correlation coefficient with WBB measurements to confirm the validity of the STC for evaluating pelvic mobility. Each movement showed a significant positive correlation.

\section{Validity of the arm stretch task}

To confirm the validity of the STC for evaluating the pelvic movement during the stretch task, Pearson's correlation

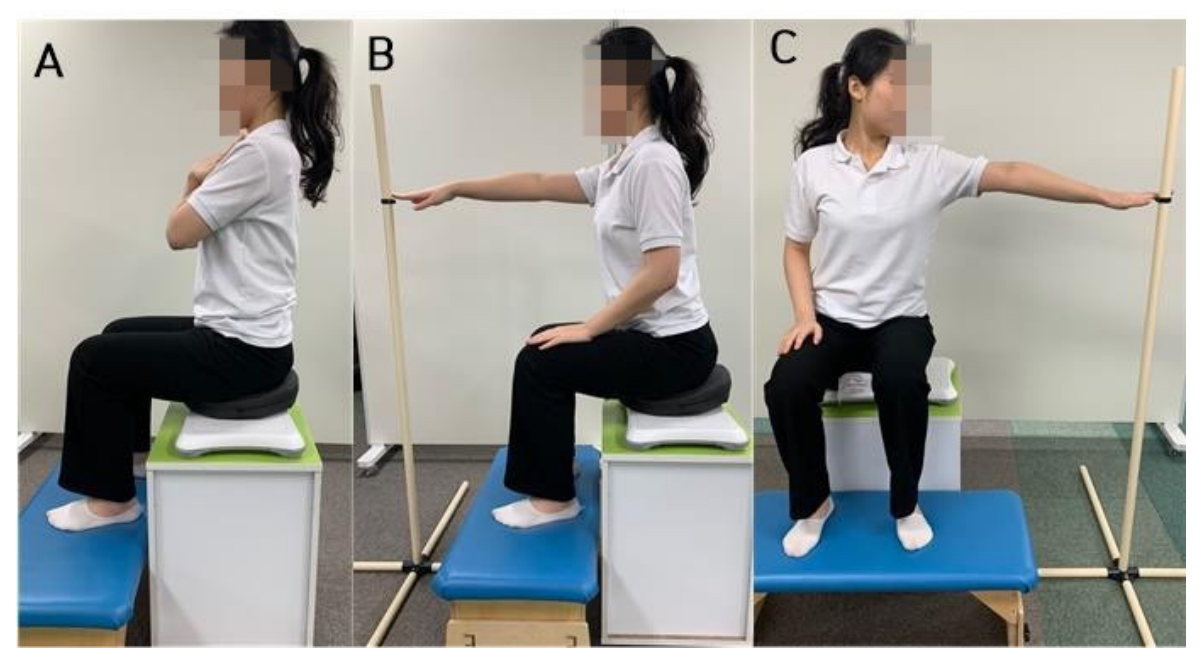

Figure 2. The setting for measuring pelvic movement $(\mathrm{A})$, during reaching task $(\mathrm{B}, \mathrm{C})$. 
Table 1. Intra-rater reliability of the Sensbalance Therapy Cushion

$(\mathrm{N}=50)$

\begin{tabular}{lcccc}
\hline Variable/division & $1 \mathrm{st}(\mathrm{n}=50)$ & 2nd $(\mathrm{n}=50)$ & Difference $(95 \% \mathrm{CI})$ & ICC $[3,1](95 \% \mathrm{CI})$ \\
\hline Tilt $\left(^{\circ}\right)$ & & & & \\
$\quad$ Anterior & $15.34(5.10)$ & $16.04(4.97)$ & $-0.70 \pm 2.82(-1.37,-0.03)$ & $0.915(0.863,0.947)^{* *}$ \\
Posterior & $10.96(3.92)$ & $11.91(4.17)$ & $-0.95 \pm 2.29(-1.49,-0.40)$ & $0.913(0.860,0.946)^{* *}$ \\
Left & $13.77(4.02)$ & $15.27(3.98)$ & $-1.5 \pm 2.31(-2.05,-0.95)$ & $0.909(0.854,0.944)^{* *}$ \\
Right & $13.98(3.74)$ & $14.87(3.69)$ & $-0.89 \pm 2.42(-1.46,-0.31)$ & $0.881(0.809,0.926)^{* *}$ \\
Proprioception $\left(^{\circ}\right)$ & & & & \\
Anterior & $7.42(4.81)$ & $5.58(3.39)$ & $1.84 \pm 5.60(-8.44,1.04)$ & $0.179(-0.032,0.490)$ \\
Posterior & $6.13(4.29)$ & $5.13(3.47)$ & $1.00 \pm 5.13(-0.22,2.22)$ & $0.243(-0.219,0.529)$ \\
Left & $4.70(3.12)$ & $4.65(3.59)$ & $0.05 \pm 4.11(-0.93,1.03)$ & $0.400(0.035,0.627)^{*}$ \\
Right & $5.89(6.44)$ & $4.74(3.67)$ & $1.16 \pm 7.00(-0.51,2.83)$ & $0.195(-0.030,0.500)$ \\
Reaction $(\mathrm{s})$ & & & & \\
Anterior & $0.97(0.31)$ & $0.87(0.27)$ & $0.10 \pm 0.36(0.01,0.19)$ & $0.341(-0.060,0.591)^{*}$ \\
Posterior & $0.93(0.21)$ & $0.86(0.17)$ & $0.06 \pm 0.22(-0.01,0.11)$ & $0.495(0.188,0.686)^{* *}$ \\
Left & $0.91(0.22)$ & $0.92(0.24)$ & $-0.002 \pm 0.24(-0.06,0.06)$ & $0.595(0.349,0.749)^{* *}$ \\
Right & $0.90(0.28)$ & $0.91(0.55)$ & $-0.01 \pm 0.51(-0.13,0.12)$ & $0.495(0.187,0.686)^{* *}$ \\
Task $\left(^{\circ}\right)$ & & & & \\
Front & $16.73(5.64)$ & $17.81(6.00)$ & $-1.08 \pm 3.53(-1.92,-0.24)$ & $0.899(0.837,0.937)^{* *}$ \\
Left & $18.11(4.80)$ & $19.61(5.14)$ & $-1.50 \pm 4.02(-2.46,-0.54)$ & $0.804(0.685,0.878)^{* *}$ \\
Right & $17.57(4.57)$ & $18.58(4.98)$ & $-1.01 \pm 3.05(-1.74,-0.29)$ & $0.886(0.817,0.929)^{* *}$ \\
\hline
\end{tabular}

Values are presented as mean (SD).

CI: confidence interval, ICC: intraclass correlation coefficient.

${ }^{*} p<0.05,{ }^{* *} p<0.01$.

Table 2. Correlation of the STC, WBB, and SEMG

\begin{tabular}{|c|c|c|c|c|}
\hline \multirow{2}{*}{ Variable } & \multicolumn{4}{|c|}{ STC tilt } \\
\hline & Anterior $\left({ }^{\circ}\right)$ & Posterior $\left({ }^{\circ}\right)$ & Left $\left(^{\circ}\right)$ & Right $\left(^{\circ}\right)$ \\
\hline \multicolumn{5}{|l|}{ WBB tilt $(\mathrm{cm})$} \\
\hline Anterior & $0.591 * *$ & - & - & - \\
\hline Posterior & - & $0.354 * *$ & - & - \\
\hline Left & - & - & $0.588 * *$ & - \\
\hline Right & - & - & - & $0.597 * *$ \\
\hline \multicolumn{5}{|l|}{ WBB reach $(\mathrm{cm})$} \\
\hline Anterior & $0.656^{* *}$ & - & - & - \\
\hline Left & - & - & $0.737 * *$ & - \\
\hline Right & - & & - & $0.534 * *$ \\
\hline \multicolumn{5}{|l|}{$\mathrm{sEMG}(\mathrm{mV})$} \\
\hline Left L3 & -0.079 & -0.074 & -0.204 & -0.136 \\
\hline Right L3 & -0.054 & -0.038 & -0.111 & -0.042 \\
\hline Left L5 & -0.040 & $-0.327 * *$ & $-0.296^{*}$ & $-0.324 * *$ \\
\hline Right L5 & -0.094 & -0.214 & $-0.031 * *$ & $-0.325^{* *}$ \\
\hline
\end{tabular}

STC: Sensbalance Therapy Cushion, WBB: Wii Balance Board, sEMG: surface electromyography. ${ }^{*} p<0.05,{ }^{* *} p<0.01$.

coefficient with the WBB measurement was shown, and the results are shown in Table 2. Each movement showed a significant positive correlation.
Correlation between pelvic mobility and lumbar static muscle activity

Table 2 shows the correlation analysis between pelvic mobility and lumbar static muscle activity. The relationship 
between lumbar static muscle activity and pelvic mobility at the left L5 level of the subjects showed a negative correlation, and was statistically significant in the measured values of posterior inclination, left lateral inclination, and right lateral inclination. The relationship between lumbar static muscle activity and pelvic mobility at the right L5 level of the subjects showed a negative correlation and was statistically significant in the measurements of the left lateral inclination and the right lateral inclination.

\section{Discussion}

This study presents the reliability and validity of a convenient measuring tool for measuring the pelvic mobility parameter to prevent low back pain among modern people who spend extended periods of time in assuming an improper sitting position, and At the same time, this study was performed to confirm the correlation between the tension of the lower back and the pelvic mobility variables caused by the prolonged sitting positions. To this end, the result measured based on the pressure sensor built into the STC and the result measured simultaneously through the WBB were compared and were verified to be reliable and valid.

As a result, among the pelvic mobility variables measured by STC, it was confirmed that the reliability of the pelvic slope was very high (excellent), the response time variable was poor (poor) to fair (fair), and was very high (excellent) in performing the arm stretch tasks.

Through STC and WBB, the results measured during the front, rear, left and right pelvic tilt evaluations and arm stretch tasks showed a high level of positive correlation and were statistically significant. Similarly, in a study by Yang et $a l$. [21], the ICC of the ground repulsion information when standing up in healthy adults using the WBB was 0.676 to 0.946, and the ICC of the ground reaction force when walking was 0.723 to 0.901 . In addition, Kim and Choi's study [22] also showed a high level of confidence in the ICC of the Limits of Stability in healthy adults using the BioRescue (RMIngénierie, Marseille, France) from 0.69 to 0.84 . In the study by Menz et al. [23]. The ICC of gait analysis information of healthy adults and the elderly using the GAITRite was 0.82 to 0.92 , showing high reliability. The reason for this result was that the measurement tools actually used were based on the pressure sensor, and the STC used in this study had high reliability and validity because the pressure sensor is built-in. As a result of this study, the inter-rater reliability was poor in evaluating the intrinsic acceptance. In this study, measurement of positional sense indicating the location of a muscle or joint was made, which is classified as a very sensitive and precise sense [24]. However, in the measurement procedure, it is thought that the measurement was embarked at a new location each time between the first and second attempt, and the error was reflected. In addition, a study by Dolan and Green [25] suggested that the crooked posture of the waist causes confusion in the intrinsic acceptance of the joint . It is thought that the subjects of this study also differed in the ability to accommodate joints due to different sitting posture habits, and this was reflected in the study results.

As a result of analyzing the correlation between pelvic mobility and lumbar static muscle activity in this study, a statistically significant negative correlation was found between the lumbar static muscle activity and the pelvic posterior slope and left and right lateral slope at both L5 levels was observed. According to Yuk et al. [26], weight bearing in the standing position without load showed a high proportioin in the L 3 and L5 segments, and in particular, the L5 segment showed higher muscle activity than the L3 segment. According to Kang's study [27], the lumbar extensor fatigue of chronic low back pain patients was higher in the order of L1, L3, and L5. In this study, it is considered that the results of the previous studies were reflected, and unlike the pelvic anterior tilt, the point that showed a clear correlation in the posterior tilt is considered to be related to the pelvic coupling force. In order for the pelvic posterior inclination to appear, the coupling forces of the vertebral, lumbar and rectus femoris muscles and the coupling forces of the rectus femoris muscles, the gluteus maximus, and the hamstring muscles are emphasized, respectively, resulting in eccentric and concentric contraction, respectively. However, it is considered that the erector spinae at L5, which shows relatively high tension, appears to interfere with the pelvic posterior tilt due to the inability to produce proper centrifugal contraction.

This study was conducted to confirm whether the reliability and validity evaluation tool is high in evaluating variables related to pelvic movement in healthy adults using the STC. As a result, it was possible to confirm more than fair reliability in the remaining variables except for the measurement of the accommodation senses of the front, rear, and right sides and the response time of the left side.

By showing a significant correlation with WBB, the validity of he pelvic mobility measurement device was verified, a significant correlation was found between static surface electromyography results, and the possibility of being a use- 
ful device for obtaining information on lumbar tension was confirmed.

The limitation of this study was that the movement of the trunk was not accurately controlled while measuring the pelvic mobility parameters. When excessive movement is produced in an unfamiliar state when trying to move the pelvis, there is a possibility that the trunk may be compensated. Using this study as a springboard, it provides a reference point for suppressing the movement of the body, and if research is conducted for a wide range of ages and various diseases, interventional studies using visual feedback and STC can be established as highly useful treatment and evaluation equipment.

In conclusion, this study was conducted to confirm whether the STC it is a reliable and appropriate evaluation tool for evaluating variables related to pelvic movement in healthy adults. As a result, it was possible to confirm more than fair reliability in the remaining variables except for the measurement of the intrinsic acceptance of the front, rear, and right sides and the response time of the left side. By showing a significant correlation with $\mathrm{WBB}$, the validity of the pelvic mobility measurement device was verified, significant correlation was found between the static surface electromyography results, and the possibility of being a useful device for obtaining information on lumbar tension was confirmed.

\section{Conflict of Interest}

The authors declared no potential conflicts of interest with respect to the authorship and/or publication of this article.

\section{References}

1. Kang SY, Kim SH, Ahn SJ, Kim YH, Jeon HS. A comparison of pelvic, spine angle and buttock pressure in various cross-legged sitting postures. Phys Ther Korea 2012;19:1-9.

2. Kim HH. The effects of spinal stabilization exercise on lumbar paraspinal and abdominal muscles function for patients with the chronic low back pain. Korea Sport Res 2007;18:135-45.

3. Panjabi M, Yamamoto I, Oxland T, Crisco J. How does posture affect coupling in the lumbar spine? Spine 1989;14:1002-11.

4. Youdas JW, Garrett TR, Harmsen S, Suman VJ, Carey JR. Lumbar lordosis and pelvic inclination of asymptomatic adults. Phys Ther 1996;76:1066-81.

5. Juhl JH, Ippolito Cremin TM, Russell G. Prevalence of frontal plane pelvic postural asymmetry - part 1. J Am Osteopath Assoc 2004;104:411-21.

6. Sahrmann SA. Does postural assessment contribute to patient care? J Orthop Sports Phys Ther 2002;32:376-9.
7. Lee KW. Therapeutic exercise in low back pain. J Korean Acad Rehabil Med 1995;19:203-8.

8. Thomas WM. The superficial back line. In: Myers TW, editor. Anatomy trains. 2nd ed. Edinburgh: Churchill Livingstone/Elsevier; 2009. p. 73-95.

9. Tateuchi H, Tsukagoshi R, Fukumoto Y, Akiyama H, So K, Kuroda $\mathrm{Y}$, et al. Pelvic instability and trunk and hip muscle recruitment patterns in patients with total hip arthroplasty. J Electromyogr Kinesiol 2013;23:151-8.

10. Cho KJ, Kim YT, Seo B, Shin J. Radiological evaluation and classification of adult spinal deformity. J Korean Orthop Assoc 2016;51:1-8.

11. Seo D, Lee Y, Lee D, Kim S. The effect of a six-week sling exercise on flexibility, balance, muscular strength, pelvic tilt angle in age of 20 woman low back pain. J Korean Soc Integr Med 2013;1:1-12.

12. Holmes JD, Jenkins ME, Johnson AM, Hunt MA, Clark RA. Validity of the Nintendo $\mathrm{Wii}^{\circledR}$ balance board for the assessment of standing balance in Parkinson's disease. Clin Rehabil 2013; 27:361-6.

13. Martinho NM, Silva VR, Marques J, Carvalho LC, Iunes DH, Botelho $\mathrm{S}$. The effects of training by virtual reality or gym ball on pelvic floor muscle strength in postmenopausal women: a randomized controlled trial. Braz J Phys Ther 2016;20:248-57.

14. Ahmad I, Noohu MM, Verma S, Azharuddin M, Hussain ME. Validity and responsiveness of balance measures using PedaloßSensomove balance device in patients with diabetic peripheral neuropathy. J Clin Diagn Res 2019;13:YC01-YC04.

15. Laessoe U, Svendsen AW, Christensen MN, Rasmussen JR, Gaml AS. Evaluation of functional ankle instability assessed by an instrumented wobble board. Phys Ther Sport 2019;35:133-8.

16. Lesimple C, Fureix C, Biquand V, Hausberger M. Comparison of clinical examinations of back disorders and humans' evaluation of back pain in riding school horses. BMC Vet Res. 2013;9:209.

17. Ambroz C, Scott A, Ambroz A, Talbott EO. Chronic low back pain assessment using surface electromyography. J Occup Environ Med 2000;42:660-9.

18. Kim GE, Yun DU, An YJ, Park DS, Ham JH. Reliability and validity of new evaluation methods using static surface electromyography in persons with neck pain. Phys Ther Rehabil Sci 2019;8:1-7.

19. Park DS, Lee DY, Choi SJ, Shin WS. Reliability and validity of the Balancia using Wii balance board for assessment of balance with stroke patients. J Korea Acad Industr Coop Soc 2013;14: 2767-72.

20. Fleiss JL. Reliability of measurement. In: Fleiss JL, editor. The design and analysis of clinical experiments. New York (NY): Wiley; 1989. p. 1-23.

21. Yang SR, Oh YR, Jeon YR, Park DS. Test-retest reliability of sit-to-stand and gait assessment using the Wii balance board. Phys Ther Korea 2016;23:40-7.

22. Kim JH, Choi BR. Intra- and inter-rater reliability of BioRescue. J Korea Contents Assoc 2018;18:348-52.

23. Menz HB, Latt MD, Tiedemann A, Mun San Kwan M, Lord SR. Reliability of the GAITRite walkway system for the quantification of temporo-spatial parameters of gait in young and older people. Gait Posture 2004;20:20-5.

24. Kim MC, Kim NJ, Lee MS, Moon SR. Validity and reliability of 
the knee joint proprioceptive sensory measurements using a smartphone. J Korean Soc Phys Med 2015;10:15-23.

25. Dolan KJ, Green A. Lumbar spine reposition sense: the effect of a 'slouched' posture. Man Ther 2006;11:202-7.

26. Yuk GC, Park RJ, Lee HY, Lee MH, Lee JH, Kuk JS, et al. The effects of baby carrier and sling in muscle activation of trunk, low extremity and foot pressure. J Korean Soc Phys Med 2010;5:22331.

27. Kang MH. The analysis of lumbar extensor fatique during trunk exercise in low back pain patients. Kor J Phys Edu 2011;20: 1085-93. 